\title{
LE DOSAGE DE L'AXÉROPHTOL DANS LES PRODUITS ALIMENTAIRES VITAMINISÉS DESTINÉS AUX ANIMAUX
}

\author{
A. C. FRANÇOIS, Andrée PIHET. \\ Service de Biochimie et de Nutrition, \\ Centre national de Recherches zootechniques, Jouy-en-Josas.
}

\begin{abstract}
SOMMAIRE
La séparation de la vitamine A est effectuée dans les aliments et les composés minéraux après

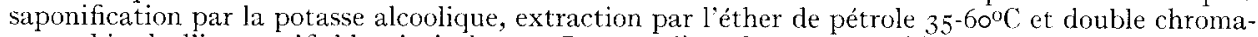
tographie de l'insaponifiable ainsi obtenu. La première chromatographie, effectuée sur magnésie permet de retenir les caroténols, la vitamine étant éluéc par le mélange acétone-éther de pétrole $(\mathrm{I}+9)$. La seconde chromatographie, effectuée sur une colonne d'alumine contenant $\mathrm{I}, 5 \mathrm{p}$. 100 d'humidité sépare les carotènes, élués par le mélange acétone-hexane $(2+98)$, de la vitamine $A$, que l'on élue par le mélange éthanol-hexane $(8+92)$. Après évaporation de l'éluat à basse température, la vitamine A est dosée au moyen de la réaction de Carr et Price (étude cinétique de la réaction).

On peut ainsi doser moins de 2 uI de vitamine A par gramme de produit. Le taux moyen de recouvrement observé pour différents aliments et contenant de I, 2 à 9,3 uI par gramme est de l'ordre de 93 p. Ioo (extrêmes : 86 à I04 p. 100). L'étude des spectres confirme la validité de la méthode proposée. Cette méthode peut être appliquée sans chromatographie aux composés minéraux.
\end{abstract}

Le dosage physico-chimique de la vitamine $\mathrm{A}$ dans les produits alimentaires vitaminisés présente deux difficultés principales qui sont: d'une part la concentration souvent très faible de la vitamine A (de l'ordre de I mcg/g) et, d'autre part, la présence de substances dont les propriétés interfèrent avec celles du rétinol. Les pigments caroténoïdes appartiennent notamment à ce groupe de substances qui perturbent soit la colorimétrie, soit la spectrophotométrie ultra-violette. En outre, dans les produits contenant la vitamine A stabilisée par certains types d'enrobage, il n'est plus possible d'extraire directement la substance active au moyen d'un solvant ; les méthodes proposées par exemple par SERVIGNE et al. (I95I) et par COOLEY (I952) ne sont plus applicables ; il est alors indispensable de saponifier le produit afin d'en libérer le rétinol. En conséquence, 1'insaponifiable contient la vitamine A sous la forme alcool. Or, le comportement chromatographique des xanthophylles, sur alumine, est beaucoup plus proche de celui de la vitamine A alcool que de celui de la vitamine A ester, et ce fait complique la séparation des deux types de substances.

Aux États-Unis, 1'A. O. A. C. s'est préoccupée du dosage de la vitamine A dans les aliments composés. COOLEy (I953) a proposé l'extraction du rétinol par l'hexane, après saponification, puis la chromatographie de l'extrait sur magnésie. L'élution par le mélange hexane-acétone permet de retenir les xanthophylles. Dans ces conditions, la vitamine $A$ et le carotène sont élués ensemble ; une correction tenant compte de la 
présence de pigment est alors nécessaire. Toutefois, le même auteur (CoOLEY I954) a proposé une technique chromatographique permettant de séparer l'axérophtol du carotène, mais les essais d'application de cette méthode ont été décevants. Ultérieurement, 1'A. O. A. C. a proposé des améliorations de cette méthode (PARRISH, I955, I956, I957, I959), mais aucune ne permet d'obtenir un éluat dépourvu de pigments caroténoïdes. C'est la raison pour laquelle a été étudiée indépendamment la méthode décrite ci-dessous, qui s'inspire à la fois de l'extraction et de la chromatographie sur magnésie proposée par COOLEY (I954) et de la technique de chromatographie sur alumine de Thompson, Ganguly, et Kon (I949).

Le principe en est le suivant : après saponification l'extrait est chromatographié sur une colonne de magnésie, qui retient les xanthophylles et permet d'éluer la vitamine $A$ accompagnée des carotènes. Les conditions expérimentales fixées ci-après permettent d'éluer cette fraction en totalité avec $50 \mathrm{ml}$ d'acétone-hexane $(\mathrm{I}+9)$. Cet éluat est évaporé, et le résidu, repris dans l'hexane, est chromatographié à son tour sur une colonne d'alumine contenant I,5 p. Ioo d'humidité. Le carotène est élué par un mélange de 2 p. Ioo d'acétone et de 98 p. Ioo d'hexane. La séparation est ainsi visuelle et, dès que la bande de carotène est passée, on élue la vitamine $A$ par un mélange contenant 8 p. Ioo d'éthanol et 92 p. Ioo d'hexane. Il est inutile de suivre la chromatographie à l'aide d'une lampe ultra-violette ; la quantité d'éluant indiquée permet en effet une élution totale de la vitamine.

Les éluats correspondant à des aliments très riches en farine de luzerne déshydratée et en maïs peuvent être encore légèrement colorés en jaune. Une correction est alors nécessaire. Celle qui a été préconisée par RAOUL et JANOT (I94I) convient bien. Ia présente méthode a été appliquée depuis I955 dans notre laboratoire. Plus tard, MORGAREIDGE, ICKEN et GARY (I957) ont signalé qu'une méthode analogue leur permettait d'obtenir des spectres et des recouvrements satisfaisants.

\section{MATÉRIEL, ETT MÉTHODES}

\section{A. - RÉactifs NÉCESSAIRES}

Acétone R. P.;

Éthanol 95 p. 100;

$n$-hexane;

Ether de pétrole distillé entre 35 et $60^{\circ} \mathrm{C}$;

Alumine Merck pour chromatographie (conditionnée à I,5 p. Ioo d'humidité) ;

Magnésie Sea-Sorb 43 ou 53 (Westswaco chlorine products);

Hyflo-Supercel ;

Réactif de CARr et PRICE;

Chloroforme lavé et distillé sur $\mathrm{CaCl}_{2}$.

Anhydride acétique RP;

Sulfate de sodium anhydre;

Éluant $A: 2$ p. 100 d'acétone -98 p. 100 d'hexane $(\mathrm{v} / \mathrm{v})$;

Éluant C: 8 p. 100 d'éthanol - 92 p. Ioo d'hexane (v/v).

$$
\text { B. - Matériel }
$$

Erlenmeyer de $300 \mathrm{ml}$ rodé avec réfrigérant à reflux; ampoule à décanter de $500 \mathrm{ml}$; petits cristallisoirs à bec ; dessicateur à vide ou appareil de distillation sous-vide ; électrocolorimètre (type Meunier par exemple, avec cuve de $25 \mathrm{~mm}$ de traversée et filtre 360 ).

Toutes les opérations doivent être effectuées à l'abri de la lumière. 


\section{C. - DOSAGE \\ T. - Cas des Aliments composés}

a) Saponification et Extraction.

Peser 5 ou $10 \mathrm{~g}$ d'aliment dans un erlenmeyer rodé de $300 \mathrm{ml}$. Ajouter $60 \mathrm{ml}$ d'alcool et $\mathrm{I} 5 \mathrm{ml}$ d'une solution de KOII à $50 \mathrm{p}$. I00. Saponifier au bain-marie à reflux pendant 30 minutes. Refroidir. 'Transvaser dans une ampoule à décanter de $25^{\circ}$ ou de $500 \mathrm{ml}$. Ajouter $60 \mathrm{ml}$ d'eau distillée. Extraire par 3 fois $50 \mathrm{ml}$ d'éther de pétrole ; laver les extraits réunis à l'eau distillée, évaporer partiellement et transvaser dans une fiole jaugée de $100 \mathrm{ml}$ contenant to $\mathrm{ml}$ d'acétone. Introduire du sulfate de sodium anhydre $(5 \mathrm{~g})$ dans la fiole; agiter et laisser reposer un moment.

b) Chromatographie.

$\mathrm{I}^{0}$ Sur Magnésie. -.. Chromatographier $50 \mathrm{ml}$ de cette solution sur une colonne de $4 \mathrm{~cm}$ de hauteur et $22 \mathrm{~mm}$ de diamètre faite d'un mélange à parties égales, en poids, de magnésie et d'hyflosupercel (tasser en s'aidant du vide).

Je carotène et la vitamine A sont élués en s'aidant du vide - (durée 15 minutes au maximum), avec un mélange d'acétone et d'éther de pétrole $(\mathrm{I}+9)(50 \mathrm{ml}$ sont généralement suff. sants); les xanthophylles restent sur la colonne. L'éluat est évaporé au bain-marie puis sous vide (dans un dessicateur, à froid ou en chauffant modérément sous azote) (MulLER, I949), et repris par 5 ou to ml d'hexane suivant la teneur du prcduit.

$2^{\circ}$ Sur alumine. - Préparer une colonne d'alumine (1,5 p. Ioo d'humidité, Serrvigne et al, 195I) de 3 à $4 \mathrm{~cm}$ de hauteur (tube de $\mathrm{I} 3 \mathrm{~cm}$ de haut et $\mathrm{I}, 3$ de diamètre intérieur prolongé d'un tube de $5 \mathrm{~cm}$ de hauteur et $0,4 \mathrm{~cm}$ de diamètre intérieur).

Un tampon de coton supporte la colonne d'alumine. Celle-ci est faite en versant dans le tube une suspension d'alumine dans l'hexane ( $4 \mathrm{~g}$ environ dans ro $\mathrm{ml}$ d'hexane). Les meilleurs résultats sont obtenus quand la colonne entière est obtenue en versant la suspension en une seule fois (ТномРson, GANGULY, KON, 1959). Laisser s'écouler l'hexane et affaiblir le pouvoir asdorbant en versant 2 à $3 \mathrm{ml}$ d'éluant $\mathrm{C}$ suivi par $5 \mathrm{ml}$ d'hexane quand la colonne est prête à être utilisée.

La solution à traiter est alors versée sur la colonne; elle est suivie par $20 \mathrm{ml}$ d'éluant $\mathrm{A}$ qui éluent les carotènes (principalement le $\beta$-carotène). Lorsque les pigments jaunes ont été élués, le réceptcur est alors changé et la vitamine $\Lambda$ alcool est éluée avec $20 \mathrm{ml}$ environ d'éluant $\mathrm{C}$ (40 $\mathrm{ml}$ si la teneur présumée est forte). La neéthode de séparation est ainsi purement visuelle et n'exige pas l'utilisation d'une lampe ultra-violette pour suivre la fluorescence de la vitamine $\mathrm{A}$.

$A$ aucun moment on ne doit se servir d'aspiration et les éluants doivent filtrer lentement au travers de la colonne. Les colonnes ne sont utilisées qu'une fois.

L'éluat contenant la vitamine A est évaporé au bain-marie, puis sous vide ou sous azote. It est repris par le chloroforme et transvasé dans une fiole jaugée de 5 à Io $\mathrm{ml}$.

La mesure est faitc à l'électrophotomètre Meunier en utilisant la réaction de CARr et PRICE $(0,5$ d'extrait + I goutte d'anhydride acétique $+2 \mathrm{ml}$ de réactif de CARR et PRICE). Dans ce cas, la quantité de vitamine $\mathrm{A}$ doit être d'environ I à I, $5 \mathrm{uI}$ dans la cuve. On suit la cinétique de la réaction pendant deux minutes en effectuant des lectures à I $5 \mathrm{~s}, 30 \mathrm{~s}, 45 \mathrm{~s}, \mathrm{I} \mathrm{mn,} \mathrm{I} \mathrm{mn} 30 \mathrm{~s}, 2 \mathrm{mn}$, après l'addition du réactif de CARR et PRICE. On extrapole la courbe au temps $O$. Pour la spectrophotométric ultra-violette, on reprend par du cyclohexane ou de l'isopropanol.

\section{II. - Cas des composés minéraux et des concentrats}

Dans le cas des composés minéraux et des concentrats, la chromatographie est souvent inutile. En effet, beaucoup de ces produits sont suffisamment concentrés et ne contiennent pas de pigments.

La méthode de saponification et d'extraction décrite précédemment reste valable. La quantité de produits à saponifier est de l'ordre de o, I $g$ pour les concentrats, de I ou $2 \mathrm{~g}$ pour les composés minéraux. Toutefois en raison de l'hétérogénéité de certains produits, il est souvent nécessaire de faire une prise d'essai de 5 ou ro g.

\section{RÉSULTATS}

La validité de la méthode décrite ci-dessus a été étudiée au moyen de tests de surcharge de quantités connues de vitamine $\mathrm{A}$ ajoutées soit à un mélange de produits minéraux, soit à plusieurs types d'aliments destinés à diverses catégories d'animaux : poussins, porcs à l'engrais, truie mère, veau. 
Les résultats obtenus au moyen de la méthode colorimétrique, sans chromatographie préalable, appliquée à un composé minéral sont groupés dans le tableau I (la vitamine A a été ajoutée au moment de la saponification, sous forme d'huile contenant environ $\mathrm{I}$ ooo $\mathrm{uI} / \mathrm{g})$.

\section{TABLEAU I}

Recouvrement de diverses quantités de vitamine $A$ ajoutées à un mélange minéral ( $2 \mathrm{~g}$ de produit).

\begin{tabular}{|c|c|c|}
\hline Quantité ajoutée & Quantité retrouvée & Recouvr \\
\hline U. I. & U. I. & $\%$ \\
\hline 62 & 68 & 110 \\
\hline 65 & 67 & 103 \\
\hline 75 & 70 & 93 \\
\hline 85 & 85 & 100 \\
\hline 147 & 147 & 100 \\
\hline 181 & 173 & 96 \\
\hline & Moyenne & $=100,3$ \\
\hline
\end{tabular}

Les recouvrements des quantités de vitamine $\mathrm{A}$ ajoutées ont été en outre vérifiés par une étude des spectres (spectrophotométre JoBIN et YvoN) : on a notamment vérifié la pureté spectrale des extraits obtenus dans différentes conditions. En effet, il importait de prouver que le dosage colorimétrique portait réellement sur un produit dont le spectre était aussi voisin que possible de celui du rétinol.

TABLEAU 2

Recourrement de la vitamine $A$ ajoutée à des aliments

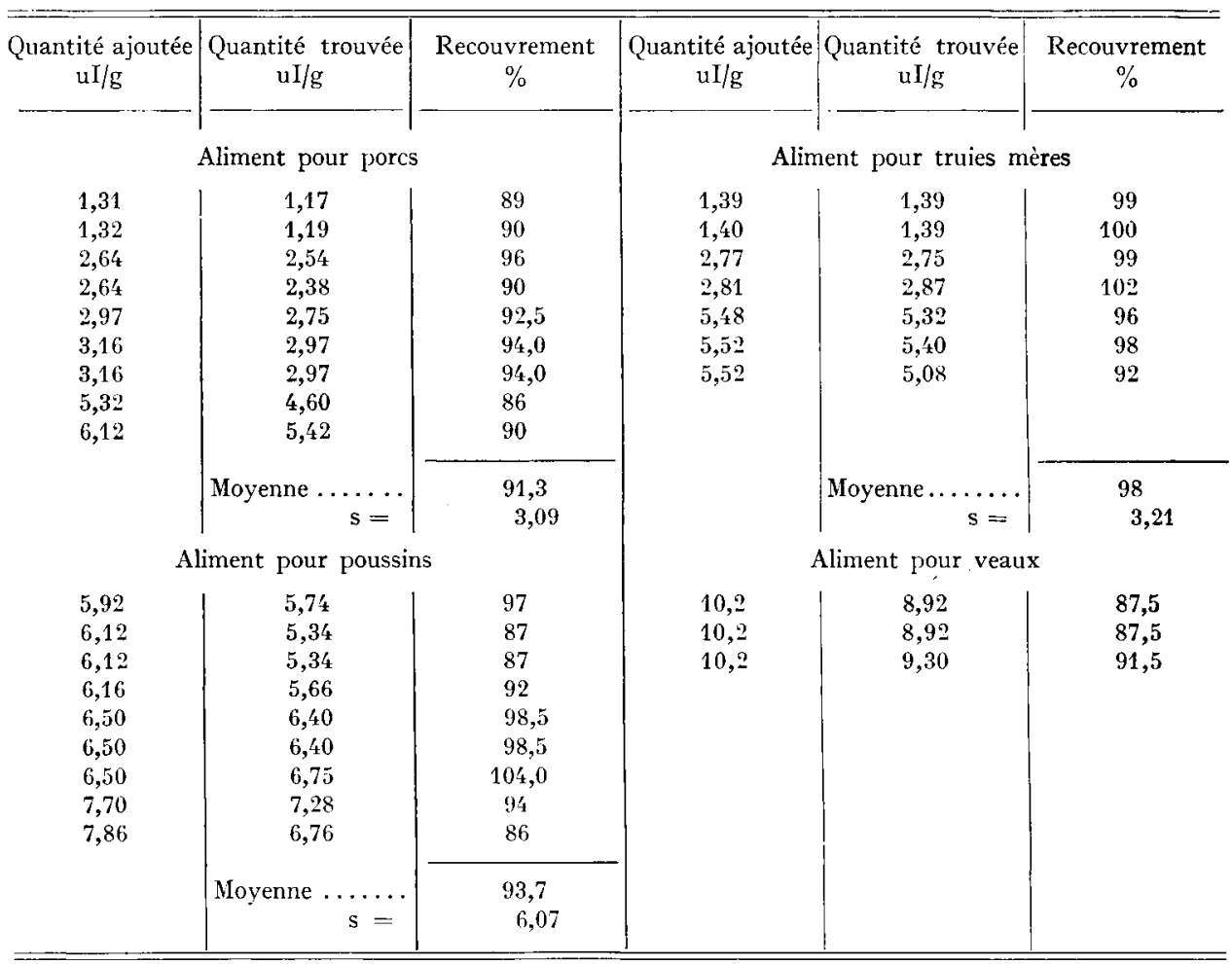


Pour ce qui concerne les surcharges effectuées à des aliments, celles-ci variaient de quantités aussi faible que I,3 uI jusqu'à Io uI par gramme. Les quantités retrouvées dans les différents types d'aliments sont indiquées dans le tableau 2.

\section{Discussion}

Les taux de recouvrement de la vitamine $\mathrm{A}$, à l'aide de la méthode décrite cidessus sont compris entre $9 I$ et Ioo $p$. Ioo. Ces taux sont très satisfaisants, compte tenu de la complexité des aliments étudiés et de la faible quantité de vitamine A dosée.

Aliments. - Pour ce qui concerne les aliments, où la double chromatographie sur magnésie et sur alumine est nécessaire, on obtient néanmoins de bons recouvrements. MoRGaREIDGE, ICKEN et Gary (r957), utilisant une méthode très voisine, indiquent qu'ils obtiennent des recouvrements qui sont, en moyenne, de 93,3 p. roo, lorsque leur méthode est appliquée à différents produits alimentaires.

Toutefois, il était nécessaire de vérifier la validité de la méthode décrite ci-dessus en contrôlant la qualité du spectre ultra-violet et en comparant les résultats quantitatifs obtenus par colorimétrie à ceux que l'on obtient par la méthode spectrophotométrique. Cette dernière méthode n'est d'ailleurs applicable qu'avec de grandes difficultés lorsque le produit contient de faibles quantités de vitamine $\mathrm{A}$, c'est-à-dire dans le cas des aliments. La figure I correspond par exemple à un aliment pour poussins contenant $6,5 \mathrm{uI}$ de vitamine A par gramme (courbe $\mathrm{I}$ ) et à un aliment pour porc contenant 3 uI par gramme (courbe 2). Malgré la double chromatographie, les courbes ne présentent qu'un épaulement dans la région 320-330 mu et ne permettent pas un dosage spectrophotométrique direct. La correction de MorTon et STubBs (1946), appliquée respectivement à l'échantillon d'aliment pour poussins et d'aliment pour

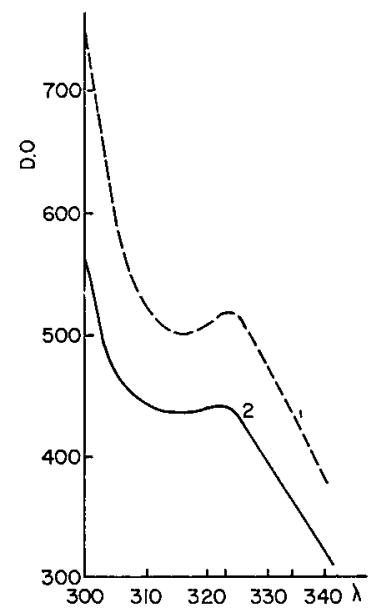

FIG. I - Spectre (dans l'isopropano' de l'insaponifiable d'aliment surchargés en vitamine $A$ (double chromatographie). - Courbe I ; aliment pour poussins. - Courbe 2 : aliments pour porcs.

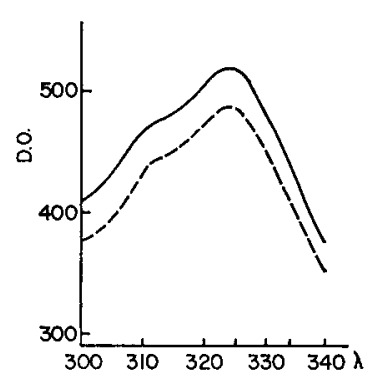

FIG. 2 - Spectre (dans l'isopropanol) de l'insaponifiable d'aliment pour veaux surchargé en vitamine $A$ (double chromatographie) (essai en double). 
porcs conduit à des recouvrements de 77 p. roo et de $97 \mathrm{p}$. Ioo, alors que la colorimétrie permet de retrouver quantitativement la surcharge dans les deux cas.

Dans le cas d'aliments relativements simples et dépourvus de substances interférentes, tels que les aliments pour veaux, la pureté du spectre est très améliorée

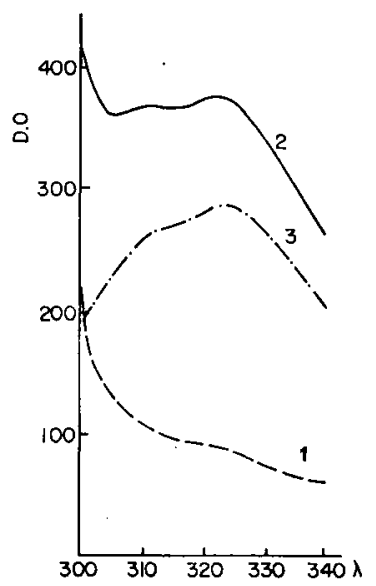

FiG.3-Comparaison des speclres (dans 'isopropanol) d'un insaponifable d'aliment pour porcs (courbe 1), et de l'insaponifiable du même aliment surchargé en vitamine $A$ (courbe 2): courbe $3=2 \ldots 1$.

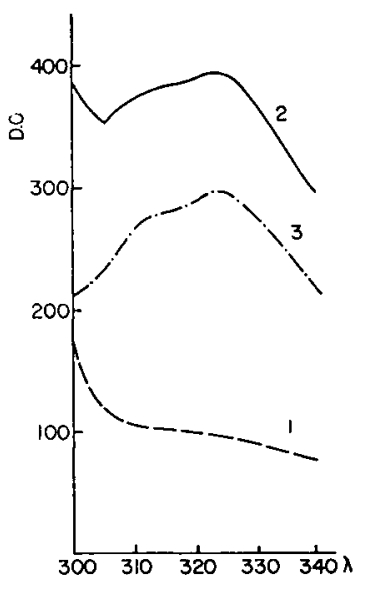

Fig.4-Comparaison des spectres (dans l'isopropanol) d'un insaponifiable d'aliment pour poussins (courbe 1), el de l'insaponifiable du même aliment surchargé en vitamine $A$ (courde 2); courbe $3=2-1$,

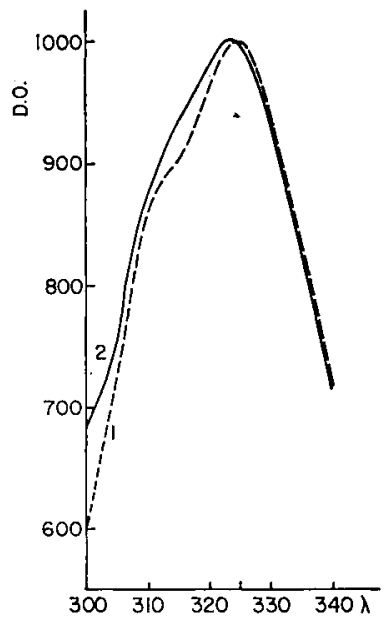

FIG. 5 - Comparaison des spectres (dans l'isopropansl) de vitamine A pure (courbe 1) et de l'insaponifiable d'un aliment surchargé après déduction de l'absortion due d' l'aliment seul (courbe 2).

(fig. 2). Malheureusement, la reproductibilité de ce spectre est mauvaise, comme le montre la même figure. L,es deux courbes sont des répétitions de dosage effectués sur des prises différentes d'un même échantillon. Une étude des spectres de différentes prises d'essai de l'aliment non surchargé en vitamine A confirme une telle variabilité, provenant vraisemblablement de l'hétérogénéité du mélange. Or, une méthode de 
choix pour calculer le taux de recouvrement consiste à déduire le spectre correspondant à 1'aliment seul, non vitaminisé, du spectre de l'aliment enrichi de vitamine. C'est ce qu'indiquent les figures 3 et 4 (les extraits de l'aliment seul et de l'aliment surchargé sont chromatographiés). Les spectres ainsi obtenus sont satisfaisants. En effet la figure 5 permet de comparer la similitude d'un spectre étalon de vitamine A pure à un spectre obtenu à partir d'un aliment pour porc (la position de maximum d'absorption est toutefois décalée vers les courtes longueurs d'onde). Par cette méthode, on observe des recouvrements compris entre go et Ioo p. Ioo, qui confirment parfaitement les résultats obtenus par colorimétrie. Ces résultats permettent ainsi de montrer que la méthode colorimétrique proposée permet de doser la vitamine $A$ dans les aliments avec une bonne sécurité.

Composés minéraux. - Ia saponification peut altérer la vitamine A notamment lorsque le milieu contient des substances capables de provoquer sa destruction, tels que certains ions métalliques, et parmi ceux-ci, notamment certains oligoéléments. Pour cette raison, la méthode de saponification ci-dessus décrite a été appliquée à une hurile vitaminée de titre connu, en présence d'un mélange de phosphates et de carbonate de calcium, de chlorure de sodium, de sels de cuivre, de cobalt et de manganèse. A deux grammes de ce mélange minéral, on ajoutait de 40 à Ioo uI par gramme, sous forme d'une huile titrant I $000 \mathrm{uI} / \mathrm{g}$. Les extraits étaient chromatographiés sur alumine I5.

L'étude de la qualité du spectre ainsi obtenu (fig. 6) montre que les caractéristiques en sont satisfaisantes $\left(\frac{\mathrm{F}: 300}{\mathrm{E} 23^{8}}=0,68\right)$ puisqu'elles sont très voisines de celles de 1'huile pure utilisée comme surcharge (à raison de roo uI par $g$ de minéral). La déformation spectrale est d'ailleurs fonction du rapport entre la quantité de vitamine A et celle du produit minéral. Par exemple, la figure 7 montre la déformation des courbes obtenues par surcharge de la vitamine A à raison de 40 uI seulement par g de mélange

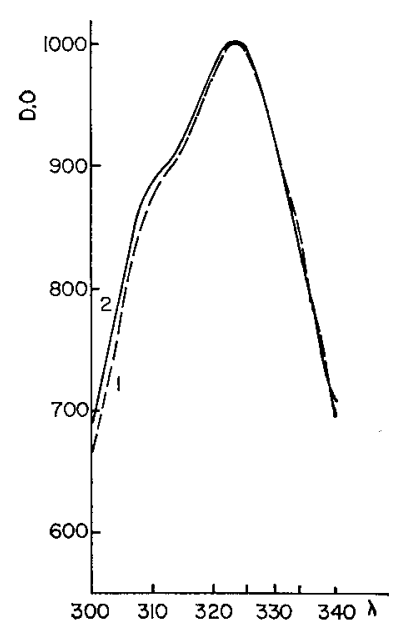

Fig. 6-Comparaison des spectres (dans l'isopropanol) de vitamine $A$ pure (courbe 1) et de l'insaponifiable d'un mélange minéral surchargé à $100 \mathrm{uI} / \mathrm{g}$ (courbe 2).

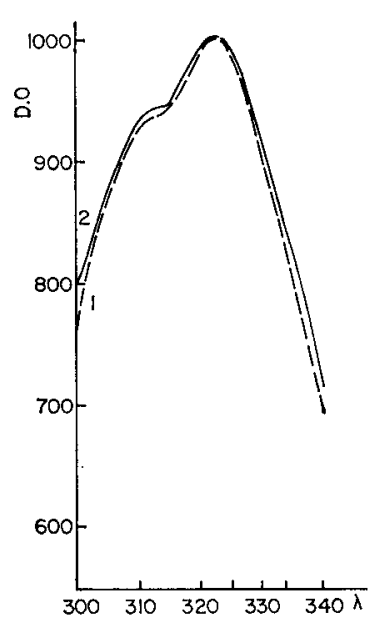

Fig. 7-Comparaison des specires (dans l'isopropanol) de vilamine $A$ pure (courbe 1) et de l'insaponifiable d'un mélange minéral surchargé à 40 $u I / g$ (courbe 2). 
minéral. Dans ce dernier cas, on a $\mathrm{E} \frac{320}{328}=0,79$. En revanche, les produits très concentrés $(2000 \mathrm{uI} / \mathrm{g}$ ) (fig. 8) permettent d'obtenir des spectres très purs. Les taux de récupération obtenus par dosage spectrophotométrique (tableau suivant) sont identiques à ceux obtenus au moyen du dosage colorimétrique proposé.

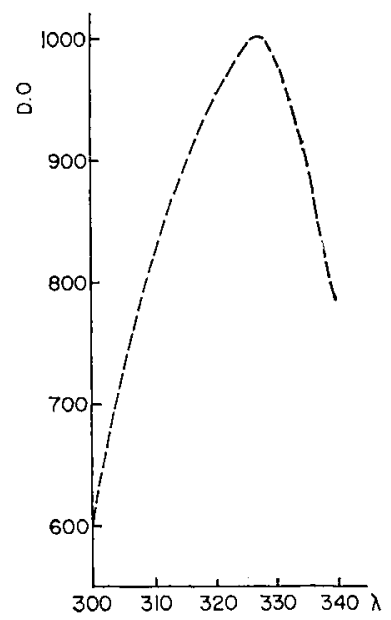

Fi.s. 8 - Spectre dx us le cyciólo ane de l'insapunifable d'un mélange minéral surchargé à 2000 ul/g

Vitamine A ajoutée
$\mathrm{uI} / \mathrm{g}$

$\overline{(a)}$

$44,1 \ldots \ldots \ldots \ldots \ldots$

$43,8 \ldots \ldots \ldots \ldots \ldots$

$100,8 \ldots \ldots \ldots \ldots \ldots$

$101,1 \ldots \ldots \ldots \ldots \ldots$
Vitamine A dosée (sans correction)

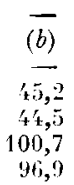

Vitamine A closée (correction
de Morton)
Recouvrement p. 100

$b \overline{\times} 100$
102
102
99,9
95,8

Recouvrement p. 100

\section{Influence de la chromatographie sur magnésie.}

Cette phase du dosage permet de séparer les caroténols qui ont un comportement chromatographique voisin de la vitamine $\mathrm{A}$ lorsqu'on utilise uniquement une colonne d'alumine. Or, certains aliments riches en maïs et en farine de luzerne deshydratée contiennent des quantités importantes de lutéine, de zéaxanthine, de violaxanthine, qui s'éluent avec la vitamine A. La chromatographie sur magnésie est une opération délicate. En effet, si elle est conduite trop lentement (plus de 15 minutes) ou sur une colonne trop importante, elle aboutit à une déformation très importante du spectre. D'autre part, les lots de magnésie sont souvent différents les uns des autres quant à leurs propriétés chromatographiques. Il est en conséquence indispensable de vérifier la qualité de tout nouveau lot.

\section{Infuence de l'extraction.}

L'hexane, 1'éther de pétrole de $\mathrm{PE} 35^{-} 60^{\circ} \mathrm{C}$ et l'éther de pétrole de $\mathrm{PE} 65-70^{\circ} \mathrm{C}$ ont été comparés. L'étude d'une huile dosant I250 uI par dosage direct a montré que 


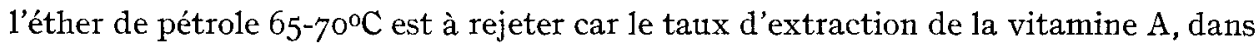
ce cas, est inférieur à celui obtenu avec de l'hexane ou de l'éther de pétrole $35^{-} 60^{\circ} \mathrm{C}$.

\section{Évaporation.}

La méthode d'évaporation peut comporter une évaporation à froid dans un dessicateur ou encore une évaporation à température réduite (inférieure à $50^{\circ} \mathrm{C}$ ), sous vide dans un évaporateur rotatif $d u$ type Craig, ou encore dans un appareil de distillation sous vide partiel avec admission d'azote, tel que celui décrit par MULLER (I946).

L'application de ces différents procédés conduit à des résultats identiques ; toutefois, des pertes de vitamine A peuvent être constatées lorsque l'évaporation à froid se prolonge en raison, par exemple, d'un vide défectueux.

Requ en mars 1960.

\section{SUMMARY}

\section{ESTIMATION OF AXEROPHTOL IN VITAMIN-ENRICHED FELDSTUFFS}

The physico-chemical determination of vitamin $A$ in vitamin-enriched feedstuffs presents two major difficulties, these are : the often very low concentration of vitamin $\Lambda$ (around I mcg/g) on the one hand, and the presence of substances whose properties interfere with those of retinol, on the other. The carotenoid pigments belong, notably, to this group of substances which upset either the colorimetry or the ultra-violet spectrophotometry. In addition, in the products containing vitamin A stabilized by various forms of coating, it is no longer possible directly to extract the active substances by means of a solvent; it is therefore essential to saponify the product in order to liberate the retinol. Consequently, the unsaponifiable residue contains the vitamin $\mathrm{A}$ in the form of alcohol. Besides, the chromatographic behaviour of the xanthophylls on alumina resembles more closely that of the vitamin A alcohol than that of the vitamin A ester, and this fact complicates the separation of the two kinds of substances.

The separation of vitamin A is effected in the feedstuffs ( 5 to $10 \mathrm{~g}$ ) and the mineral mixtures after saponification with alcoholic potassium hydroxide ( $15 \mathrm{ml} \mathrm{KOH}$ at $50 \mathrm{p} .100: 60 \mathrm{ml}$ ethanol),

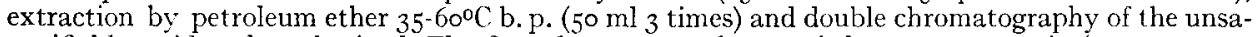
ponifiable residue thus obtained. The first chromatography, carried out on magnesia $(40 \times 12 \mathrm{~mm}$ column), enables the carotenols to be retained, the vitamin being eluted by the acetone-petroleum ether nixture $(\mathrm{r}+9)$ (50 ml generally suffice). The second chromatography, carried out on an alumina column $\left(40 \times I_{3} \mathrm{~mm}\right)$ containing 1,5 p. 100 moisture, separates the carotenes, eluted by the acetone-hexane mixture $(2+98)(20 \mathrm{ml})$, from vitamin $\mathrm{A}$, which is eluted by the ethanolhexane mixture $(8+92)(20 \mathrm{ml})$. After evaporation of the eluate at low temperature, the vitamin $\mathrm{A}$ is estimated by means of the Carr and Price reaction (kinetic study of the reaction).

Less than 2 I. U. of vitamin A per gram of product can thus be determined. The average recovery level observed for different feedstuffs containing from $\mathrm{I}, 2$ to 9,3 I. U. per gram is in the region of $93 \mathrm{p}$. 100 (extremes : 86 to 104 p. 100). A study of the spectra confirms the validity of the proposed method. The spectra obtained after deduction of that corresponding to the feedstuff alone, without vitamin, were studied in particular.

This method can be applied without chromatography to mineral mixtures.

\section{RÉFÉRENCES BIBLIOGRAPHIQUES}

Cooley M., 1951. Report on Vitamin A in mixed feeds. J. Ass. Off. Agric. Chem. Wash., 34, 370.

Cooley M., 1952. Report on Vitamin A in mixed feeds. J.Ass. Off. Agrie. Chem. Wash., 35, 706-715.

Cooley M., i 95.3. Report on Vitamin A in mixed feeds. J.Ass. Off. Agric. Chem. Wash., 36, 81 2-819.

Cooley 11., I954. Report on Vitamin A in mixed feeds. J. Ass. Off. Agric. Chem. Wash., 37, 742-748.

Meunier P., Raoul. Y., I942. Le diagnostic chimique des avitaminoses. E. Masson et Cie, Paris.

Morgareidge K., ICKeN J., GaRY H., I 957 . Jistimation of vitamin A in food following tandem column chromatography on alumina. American Chemiçal Society. Abstracts of papers presented at Miami, 1 A. 
Morton R. A., Stubbs A. L., 1946. Photoelectric spectrophotometry applied to the analysis of mixtures and Vitamin A oils. Analyst, $71,348$.

MUller P. B., I949. Vitaminbestimmung in lebensmitteln mit chemischen methoden. Travaux de Chimie Alimentaire et d'Hygiène, 40, 359-4I 5 .

PARrish D. B., I955. Report on Vitamin A in mixed feeds. J. Ass. Off. Agric. Chem. Wash., 38, 692.

Parrish D. B., Smith A. A., 1956. Report on Vitamin A in mixed feeds. J. Ass. Off. Agric. Chem. Wash., 39, I 26.

PARRISH D. B., 1957. Report on Vitamin A in mixed feeds. J. Ass. Off. Agric. Chem., Wash., 40,865.

Parrish D. B., I959. Report on Vitamin A in mixed feeds. J. Ass. Off. Agric. Chem., Wash., 42, 520.

RAOUl Y., JanOT M. M., r94x. Sur le dosage de la Vitamine A et du carotène dans le sang. Bull. Soc. Chim. Biol., 23, 78 .

Servigne M., Guírin de Montgareuil P., Pinta M., 195x. Fractionnement chromatographique et dosage de la Vitamine A. Pub. C. N.R. S.

Thompson S. Y., Ganguly J., Kon S. K., I949. The conversion of carotene to Vitamin A in the intestine. Brit. J. Niti., 3, 50. 